\title{
Relación entre el estilo personal del terapeuta, estilo de apego y factores de personalidad del terapeuta
}

Gabriel Genise ${ }^{1}$

\section{Artículo}

Material original autorizado para la publicación en la revista Psicodebate. Facultad de Ciencias Sociales. Universidad de Palermo.

Recibido 19-09-2014 | Aceptado 17-11-2014

\section{Resumen}

El objetivo de este estudio es analizar la relación entre el estilo personal del terapeuta, estilo de apego y factores de personalidad del terapeuta. Para lo cual se aplicó el cuestionario del Estilo Personal del Terapeuta (Fernández Álvarez \& García, 1998), la escala argentina de apego (Carreras, Brizzo, Gonzalez, Mele \& Casullo, 2008) y el Big Five Inventory (Castro Solano \& Casullo, 2001). La muestra del estudio estuvo compuesta por 120 psicoterapeutas de edad promedio de 36.28 años $(\mathrm{DE}=9.65)$, y los años de experiencia promedio fueron de 7.90 años $(\mathrm{DE}=8.04)$. El análisis de los resultados mostró que existe una correlación positiva, significativa y de baja intensidad entre el factor de personalidad de apertura a la experiencia y el estilo personal de involucración, una correlación negativa, significativa y de mediana-baja intensidad entre el factor de extraversión y el modo de apego no romántico ansioso, y una relación positiva significativa de baja intensidad entre el factor de personalidad neuroticismo y el modo de apego romántico ansioso.

Palabras Clave: estilo personal del terapeuta, personalidad, apego, investigación en psicoterapia. 


\section{Abstract}

The aim of this study is to analyze the relationship between the personal style of the therapist, attachment style and personality trait. It was used the personal style of the therapist questionnaire (PST - Q), the Argentinean attachment inventory and the big five inventory. The study sample consisted of 120 psychotherapists average age of 36.28 years $(S D=9.65)$, and the average years of experience was 7.90 years $(S D$ $=8.04$ ). The analysis of the results showed that there is a positive, significant and low intensity between the personality factor of openness to experience and personal style of involvement, a negative correlation, significant and of medium intensity between low extraversion factor correlation and mode not anxious romantic attachment and a significant positive relationship between the low-intensity factor neuroticism personality and how anxious romantic attachment.

Keywords: personal style of the therapist, personality, attachment, psychotherapy research. 
La investigación empírica en los últimos 50 años puso en evidencia la relevancia de las características del terapeuta en la determinación de los procesos y resultados de la psicoterapia. No se encontró hasta el momento una clara evidencia acerca de la manera en que interactúan las habilidades y características de los terapeutas en la eficacia del proceso psicoterapéutico (García \& Fernández Álvarez, 2007). En este sentido, Huppert, Gorman, Barlow y Shear (2001) refieren que la contribución de los terapeutas en el resultado de la terapia ha sido un foco de interés. Los autores refieren que la mayoría de las investigaciones se han centrado en la edad del terapeuta y en los resultados en psicoterapia. Dichos estudios tienden a concluir que la edad del terapeuta no afecta el resultado del proceso. En este sentido, Luborsky, McLellan, Goody, O'Brien y Auerbach (1985) encontraron que terapeutas con determinadas características poseen mejores resultados con una población específica de pacientes. Dichos factores incluyen características demográficas tales como edad, género, raza y religión, y habilidades de entrenamiento (título, entrenamiento, años de experiencia, creencias y características personales). En una revisión realizada por Beutler, Machado y Allstetter Neufeldt (1994) el estilo del terapeuta constituye un estado objetivo y específico de la terapia que presenta cierta estabilidad temporal. Dentro de este marco de investigaciones, surge el constructo del Estilo Personal del Terapeuta.

Se han realizado importantes investigaciones sobre éste tópico. La primera de ellas surge como parte del proyecto conjunto entre la Fundación Aiglé (Buenos Aires) y la Universidad Ramón Llull (Barcelona). En cuanto a los objetivos, se propusieron investigar la compatibilidad de estilo personal del terapeuta y las características del paciente, especialmente lo referido a la resistencia y estilo de afrontamiento.

El segundo estudio fue realizado en Santa Bárbara, California. Se contó con una muestra de 305 pacientes y 40 terapeutas, en donde se encontró que aquellos pacientes más resistentes respondieron significativamente mejor al tratamiento, interactuando con terapeutas más espontáneos en la función operativa. Los pacientes con un estilo de afrontamiento predominantemente externalizador respondieron significativamente mejor a la terapia, interactuando con terapeutas más pautados en la función operativa (García \& Fernández Álvarez, 2007).

Dentro de esta línea de investigación, se realizaron varias series de trabajos (Castañeiras, Ledesma, García \& Fernández Álvarez, 2008; Corbella, Balmaña, Fernández Álvarez, Saúl, Botella \& García, 2009; Corbella Santoña et al., 2008; Fernández Álvarez, García, Lo Bianco \& Corbela Santomá, 2003; Rial, García, Castañeiras, Gómez \& Fernández Álvarez, 2006). Un estudio correlacional entre el estilo personal del terapeuta (en adelante se nombrará EPT) y la personalidad del terapeuta publicado recientemente por Estrada Arana (2014) mostró los siguientes resultados: la escala Hs (hipocondría) correlaciona de manera significativa y negativa con el estilo instruccional, o sea que a mayor hipocondriasis se esperaría 
encontrar que la función instruccional sea más bien flexible por parte del terapeuta. La función atencional también correlacionó de manera negativa y significativa con las escalas de Paranoia, Hipomanía y la escala de validez F. Los resultados sugieren que, mientras más focalizados sean los terapeutas en la función atencional, se esperaría encontrar menor paranoia, mayor energía psicológica y menos barreras y defensas personales. Finalmente, la función operativa correlaciona en forma positiva y de manera significativa con la escala de psicastenia (Ps); es decir, en un terapeuta pautado en lo operativo, esperamos encontrar puntajes de moderados a elevados en la escala de psicastenia y obsesión.

En cuanto al estilo de apego en la figura del terapeuta existe poca información hasta el momento. La información encontrada sugiere que la calidad de la relación terapéutica, los temas que surgen en terapia y las competencias percibidas del terapeuta, se encuentran relacionadas con el estilo de apego del terapeuta. Rubino, Barker, Roth, y Fearon (2000) exploraron la ruptura de alianza en procesos psicoterapéuticos y el estilo de apego tanto de los terapeutas como de los clientes, encontrando que los terapeutas con un estilo de apego ansioso tendían a responder con un estilo menos empático, particularmente con clientes que presentaban un estilo de apego seguro.

Henry, Schacht y Strupp (1990) demostraron la importancia del estilo de apego. Los autores refieren que las representaciones internas de las relaciones pasadas de los terapeutas tienen una influencia importante en el proceso de las sesiones con los clientes y en la calidad de la alianza, al menos con algunos clientes. Un estudio muy significativo en esta temática fue el de Black, Hardy, Turpin y Parry (2005), el cual consistió en un estudio de revisión de los estilos de apego y su rol en la psicoterapia individual. La investigación consistía en relacionar el estilo de apego del terapeuta con la alianza terapéutica y la orientación teórica. El estudio arrojó como resultado que los estilos de apego de los terapeutas se encuentran significativamente asociados con la calidad percibida de las relaciones terapéuticas con los clientes y los problemas detectados en terapia. A su vez, los terapeutas que presentaron un estilo de apego seguro manifestaron tener una mejor calidad en la alianza terapéutica con sus clientes y los terapeutas que presentaron un estilo de apego inseguro manifestaron una calidad de alianza terapéutica pobre con sus clientes. El mismo estudió arrojó como resultado que los terapeutas con apego seguro presentaban mayores probabilidades de desafiar los modelos internos de relación de sus clientes que los terapeutas con un estilo de apego inseguro. Así también, los autores refieren que los estilos de apego seguro en adultos son mediados por un modelo de trabajo simple y consistente, que es comunicado a través de narrativas coherentes y colaborativas, caracterizadas por un monitoreo meta cognitivo. Por otro lado, las narrativas inconsistentes e incoherentes serían indicadores de modelos generalmente contradictorios, similares a las narrativas obtenidas en las experiencias 
con las figuras de apego en la infancia (Skourteli \& Lennie, 2011).

Corbella, Botella, Fernández Álvarez, Saúl y García (2009) comentan el estudio realizado en donde participaron la universidad Ramón Llull, la UNED, la Universidad de Salamanca, la Universidad de Belgrano y la Fundación Aiglé. Dicho trabajo tenía por objetivo estudiar cómo se desarrolla el EPT durante un período de entrenamiento de psicoterapeutas de 18 meses.

Con respecto a la relación con la variable de apego, los terapeutas que presentaron un estilo de apego inseguro, mostraron grandes niveles de rigidez en la función instruccional del EPT - C en comparación con aquellos terapeutas que presentaron un estilo de apego seguro.

En cuanto a la investigación existente sobre personalidad y apego, varios estudios reportaron (Brennan, Clark \& Shaver, 1998; Costa \& McCrae, 1992; John, Donahue $\&$ Kentle, 1991) una correlación entre el estilo de apego y las medidas del factor de personalidad. En general, las mismas muestran que un apego seguro se correlaciona moderadamente con neurotisismo y se correlaciona de manera positiva con las variables extroversión y agradabilidad. Finalmente, se correlaciona modestamente con la variable escrupulosidad y no se correlaciona con apertura. Un apego ansioso se correlaciona fuertemente con neuroticismo y no se correlaciona con apertura. La relación existente entre el apego ansioso y las otras tres variables es poco clara. El apego evitativo se correlaciona de manera modesta con las variables de extroversión y agradabilidad y no así con apertura (Noftle \& Shaver, 2006).

En la misma línea, el objetivo de este trabajo es analizar la relación entre el estilo personal del terapeuta con el estilo de apego y factores de personalidad según el modelo de los Big Five.

\section{Método}

\section{Diseño}

El presente trabajo propone un estudio no experimental de tipo transversal correlacional que utiliza técnicas del método cuantitativo. Siguiendo a Hernández Sampieri, Fernández Collado y Baptista Lucio (1997) los diseños correlacionales tienen por finalidad describir relaciones entre dos o más variables en un momento determinado. Por lo tanto, estos diseños pueden limitarse a establecer relaciones entre variables sin la necesidad de precisar sentido de causalidad.

\section{Participantes}

La muestra estuvo compuesta por 120 participantes psicoterapeutas, 102 mujeres $(85 \%)$, y 18 hombres (15\%), la edad promedio de la muestra fue 36.28 años $(\mathrm{DT}=9.65)$, y los años de experiencia promedio fueron de 7.90 años $(\mathrm{DE}=$ 
8.04). En la tabla 1 que se presenta a continuación pueden verse los estadísticos descriptivos de la muestra.

Tabla 1.

Estadísticos descriptivos

\begin{tabular}{lcccc}
\hline & $M$ & $D E$ & Mín & Máx \\
\hline Edad & 36.28 & 9.66 & 25.00 & 65.00 \\
Años de Experiencia & 7.90 & 8.04 & 0.20 & 42.00 \\
\hline
\end{tabular}

\section{Recolección de datos}

Estilo Personal del Terapeuta. Para evaluar el estilo personal del terapeuta, se utilizará el EPT-C realizado por Fernández Álvarez y García (1998). El EPT -C es un inventario autodescriptivo de 36 ítems en una escala tipo Likert (1-7) para medir las dimensiones que constituyen el constructo: Instruccional, expresiva, involucración, atencional y operativa. Se utilizó el coeficiente alfa de Cronbach para medir la validez de cada función: Instruccional $=.69$, Expresiva $=.75$, Involucración $=.75$, Atencional $=.80$ y Operativa $=.76($ Fernández Álvarez et al., 2003).

Estilo de apego. Para evaluar el estilo de apego, se utilizará la escala argentina de estilos de apego desarrollada por Casullo y Fernández Liporace (2004). Es un instrumento psicométrico autoadministrable, que consta de dos partes que evalúan el apego en dos contextos diferenciados: el de los vínculos románticos y el de las díadas no románticas. Es una escala de tipo Likert de 1 a 4 (casi nunca hasta casi siempre) (Carreras, Brizzo, Gonzalez, Mele \& Casullo, 2008). La fiabilidad total por escala y la consistencia correspondiente a cada factor se obtienen mediante el coeficiente de alfa de Cronbach. Los investigadores citados examinan la consistencia interna de cada versión de la escala (.45 y .52 respectivamente), que resultaban aceptables en virtud del bajo número de reactivos que el instrumento posee. Para la escala sobre relaciones no románticas, el análisis arrojó la presencia de tres factores que han de explicar el $45 \%$ de la varianza total, registrando aceptables índices de fiabilidad por factor (.60 a .28), teniendo en cuenta que se trata de subescalas compuestas por dos elementos (Casullo \& Fernández Liporace, 2004).

Factor de Personalidad. Finalmente se utilizará el Big Five Inventory para evaluar los estilos de personalidad. El inventario consta de 44 ítems que evalúan los cinco grandes rasgos de personalidad. Los mismos responden sobre la base de una escala con formato Likert con 5 opciones de respuestas. La escala ha demostrado una validez concurrente con otros instrumentos reconocidos que evalúan la personalidad. Para verificar la homogeneidad de cada una de las escalas del BFI se ha realizado un estudio de fiabilidad, calculando el coeficiente alfa de Cronbach 
para cada una de las mismas. Para el factor de extraversión se obtuvo un valor .67, para el factor afabilidad .67, para consciencia .74, para el factor neuroticismo .68 y para el factor apertura se obtuvo un valor de .72. Se podría decir, por los datos obtenidos, que el BFI es un instrumento confiable verificándose la homogeneidad de cada una de las escalas que la componen (Castro Solano \& Casullo, 2001).

En cuanto al procedimiento de recolección de datos, los participantes completaron los cuestionarios de manera online, informando previamente su consentimiento a participar de la investigación. El procedimiento se ajustó a los requerimientos de la International Test Commission (International Test Commission, 2014).

\section{Resultados}

Análisis de correlaciones entre los factores de personalidad y el estilo personal del terapeuta

Con el propósito de estudiar la relación entre los factores de personalidad, evaluados a partir del BFI, y el estilo personal del terapeuta, a partir del EPT-C, se llevó a cabo una correlación producto-momento de Pearson entre las medidas. Este análisis se realizó debido a que las distribuciones de las sub-escalas mostraron no diferenciarse de las de una distribución normal asintótica.

En la tabla 2 pueden observarse los valores de correlación entre las subescalas de personalidad y de estilo personal del terapeuta. El análisis realizado muestra únicamente una correlación positiva, significativa y de baja intensidad entre el factor de personalidad de Apertura a la Experiencia y el estilo personal de Involucración $(r=.23, p<.05)$.

Tabla 2.

Correlaciones entre las sub-escalas de BFI y EPT-C.

\begin{tabular}{lccccc}
\hline & Atencional & Expresiva & Instruccional & Involucración & Operativa \\
\hline Extroversión & -.157 & -.003 & .017 & .057 & -.076 \\
Agradabilidad & .005 & -.027 & -.049 & .128 & -.043 \\
Responsabilidad & .085 & -.051 & -.045 & -.131 & -.039 \\
Neuroticismo & .066 & -.022 & .001 & .126 & .112 \\
Ap. Experiencia & -.149 & .058 & -.100 & $.226^{*}$ & -.078 \\
\hline${ }^{*} p<.05$ & & & & &
\end{tabular}

Análisis de correlaciones entre los estilos de apego y el estilo personal del terapeuta.

Con el propósito de estudiar la relación entre el estilo de apego, evaluado con el EAP, y el estilo personal del terapeuta, evaluado a partir del EPT-C, se llevó a cabo un análisis de correlación simple por rangos ordenados de Spearman. Este 
análisis se realizó dado que las distribuciones de los valores de los estilos de apego mostraron diferencias significativas con la distribución normal asintótica, por lo cual se selecciona esta prueba estadística no paramétrica. El análisis de correlaciones realizado no muestra asociaciones significativas entre las sub-escalas de los distintos tipos de apego y los diferentes estilos personales de terapeutas.

En la tabla 3 se observan los valores de correlación entre las sub-escalas de apego y de estilo personal del terapeuta.

Tabla 3.

Correlación entre EAP y EPT-C.

\begin{tabular}{|c|c|c|c|c|c|}
\hline & Atencional & Expresiva & Instruccional & Involucración & Operativa \\
\hline $\begin{array}{l}\text { Apego no romántico: } \\
\text { Temeroso Evitativo }\end{array}$ & -.076 & -.150 & .030 & -.056 & -.126 \\
\hline $\begin{array}{l}\text { Apego no romántico: } \\
\text { Ansioso }\end{array}$ & .145 & -.075 & .038 & .017 & .073 \\
\hline $\begin{array}{l}\text { Apego no romántico: } \\
\text { Seguro }\end{array}$ & -.088 & -.051 & -.065 & -.170 & -.124 \\
\hline $\begin{array}{l}\text { Apego romántico: } \\
\text { Temeroso evitativo }\end{array}$ & .001 & -.137 & .037 & -.048 & -.057 \\
\hline $\begin{array}{l}\text { Apego romántico: } \\
\text { Ansioso }\end{array}$ & -.030 & -.063 & .032 & -.023 & .004 \\
\hline $\begin{array}{l}\text { Apego romántico: } \\
\text { Seguro }\end{array}$ & .090 & .022 & .006 & .125 & -.016 \\
\hline
\end{tabular}

Análisis de correlaciones entre los estilos de apego y los factores de personalidad.

Con el objetivo de estudiar la relación entre los estilos de apego, evaluados a partir del EAP, y los factores de personalidad, evaluados a partir del BFI, se llevó a cabo un análisis de correlación simple por rangos ordenados de Spearman. Al igual que el análisis anterior, este análisis se realizó debido a que las distribuciones de los valores de las sub-escalas de apego mostraron distribuciones que se alejaron significativamente de la distribución normal.

El análisis de correlaciones realizado muestra una correlación negativa, significativa y de mediana-baja intensidad entre el factor de extraversión y el modo de apego no romántico: ansioso $(\rho=-.30, p<.01)$. También muestra una relación positiva, significativa y de baja intensidad entre el factor de apertura a la experiencia y el modo de apego romántico: seguro $(\rho=.25, p<.01)$, y finalmente una correlación positiva, significativa y también de baja intensidad, entre el factor de personalidad de neurotisismo y el modo de apego romántico: ansioso $(\rho=.22, p<.05)$.

En la tabla 4 pueden observarse los valores de correlación entre las subescalas de apego y de personalidad. 
Tabla 4.

Correlación entre EAP y BFI.

\begin{tabular}{|c|c|c|c|c|c|}
\hline & $\begin{array}{l}\frac{.0}{0} \\
\frac{0}{0} \\
\frac{0}{0} \\
\frac{0}{ \pm} \\
\text { ய }\end{array}$ & $\begin{array}{l}\overline{0} \\
\frac{\pi}{0} \\
\frac{\overline{0}}{\overline{0}} \\
\frac{\pi}{0} \\
\frac{\pi}{0} \\
\overline{0} \\
\frac{\pi}{4}\end{array}$ & 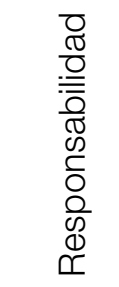 & 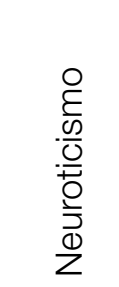 & 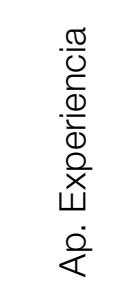 \\
\hline Apego no romántico: Temeroso Evitativo & -.081 & .112 & -.060 & .148 & -.057 \\
\hline Apego no romántico: Ansioso & $-.298^{\star \star}$ & .091 & .144 & .169 & -.006 \\
\hline Apego no romántico: Seguro & -.047 & .019 & -.018 & -.069 & .003 \\
\hline Apego romántico: Temeroso evitativo & -.037 & .111 & -.043 & .053 & -.116 \\
\hline Apego romántico: Ansioso & -.103 & .085 & .054 & $.221^{\star}$ & -.088 \\
\hline Apego romántico: Seguro & .029 & .018 & .057 & .021 & $.247^{\star *}$ \\
\hline
\end{tabular}

\section{Discusión}

Comparando estudios de similares características, se observan algunas semejanzas con estudios anteriores: El factor de personalidad Neurotisismo correlaciona positivamente con el estilo de apego ansioso (Corbella, Botella, Fernández Álvarez, Saúl \& García, 2009; Noftle \& Shaver, 2006). El estilo de apego ansioso se caracteríza por inseguridades; el neurotisismo, por otro lado, también se encontraría relacionado con inseguridades. El estilo de apego ansioso se encontraría relacionado con la faceta de depresión y vulnerabilidad del neurotisismo. De acuerdo con los resultados obtenidos en el presente estudio, existe también una correlación negativa entre el factor de extraversión y el modo de apego no romántico ansioso. Esto sostendría la hipótesis de que las personas que presentan un estilo de apego ansioso podrían encontrarse interesados en relacionarse socialmente; sin embargo, no se sienten lo suficientemente confiados para realizarlo. Estos resultados han sido obtenidos en otros estudios (Shaver \& Brennan, 1992).

Finalmente, la información obtenida sugiere que existe una correlación positiva entre el factor de apertura a la experiencia, estilo de apego seguro y estilo personal de involucración. Estos resultados apoyarían la hipótesis de que los terapeutas que presentan un estilo personal de involucración, presentarían características constituyentes tales como: imaginación, creatividad, curiosidad tanto por el medio externo como por el interno.

El presente estudio tuvo como disparador el trabajo escrito por García y Fernández Álvarez (2007) en donde surgía la pregunta de si el estilo personal del terapeuta es un estilo de personalidad. A la vista de los resultados observados, 
podemos responder que el EPT presenta estabilidad en el tiempo, sin embargo es necesario continuar estudiando el tema en futuras líneas de investigación que permitan dar una respuesta a estos interrogantes.

\section{Limitaciones}

El presente estudio posee una serie de limitaciones a tener en cuenta. En la muestra participaron más mujeres que hombres, el género se encuentra relacionado a la calidad de las relaciones. Por otro lado, el número de la muestra debería ampliarse para poder tener datos más contundentes, si bien los obtenidos son muy similares a estudios que lo preceden. 


\section{Referencias}

Beutler, L., Machado, E. \& Allstetter Neufeldt, S. (1994). Therapist Variables. En A. Bergin \& S. Gardfield (Eds.), Handbook of Psychotherapy and Behavior Change (pp. 229-269). New York: Wiley.

Black, S., Hardy, G., Turpin, G. \& Parry, G. (2005). Self-reported attachment style and therapeutic orientation of therapists and their relationship with reported general alliance quality and problem in therapy. Psychology and Psychotherapy: Theory, research and practice, 78, 363-377.

Brennan, K., Clark, C., \& Shaver, P. (1998). Self-report measurement of adult attachment: An integrative overview. En J. R. Simpson (Ed.), Attachment theory and close relationships (pp. 47-57). New York: Guilford Press.

Carreras, M., Brizzo, A., Gonzalez, R., Mele, S., \& Casullo, M. M. (2008). Los estilos de apego en los vínculos románticos y no románticos. Estudio comparativo con adolescentes argentinos y españoles. Revista Iberoamericana de Diagnóstico y Evaluación Psicológica, 25(1), 107-124.

Castañeiras, C., Ledesma, R., García, F., \& Fernández Álvarez, H. (2008). Evaluación del Estilo Personal del Terapeuta: Presentación de una versión abreviada del Cuestionario EPT-C. Terapia Psicológica, 26(1), 1-13.

Castro Solano, A. \& Casullo, M. M. (2001). Rasgos de personalidad, bienestar psicológico y rendimiento académico en adolescentes argentinos. Interdisciplinaria, 18(1), 65-85.

Casullo, M. M. \& Fernández Liporace, M. (2004). Evaluación de los estilos de apego en adultos. Anuario de investigaciones, 7, 183-192.

Corbella, S., Balmaña, N., Fernández Álvarez, H., Saúl, L., Botella, L., \& García, F. (2009). Estilo personal del terapeuta y teoría de la mente. Revista Argentina de Clínica Psicológica, 28(2) 125-133.

Corbella, S., Botella, L., Saúl, L. A., García, F. \& Fernández Álvarez, H. (2009, junio) Developing the therapist's personal style. Trabajo presentado en la 40th Annual Meeting of The Society for Psychotherapy Research, Santiago de Chile, Chile. 
Corbella, S., Fernández Álvarez, H., Saúl Gutierrez, L., García, F., Botella, L. \& García del Cid, L. B (2008) Estilo personal del terapeuta y dirección de intereses. Aportes del Psicología 26(2), 281-289.

Costa, P. \& McCrae, R. (1992). Revised NEO personality Inventory (NEO PI $R$ ) and NEO five factor inventory (NEO FFI) professional Manual. Odessa: Psychological Assessment Resources.

Estada Arana, B. (2014) Correlaciones entre el estilo personal del terapeuta y escalas clínicas del MMPI II. Revista Argentina de Clínica Psicológica, 23(2), $153-162$.

Fernández-Álvarez, H. \& García, F. (1998). El estilo personal del terapeuta: Inventario para su evaluación. En S. Gril, A. Ibáñez, I. Mosca \& P. L. R. Sousa (Eds.), Investigación en Psicoterapia (pp. 76-84). Pelotas: Educat.

Fernández Álvarez, H., García, F., Lo Bianco, J. \& Corbella, S. (2003). Assessment Questionnaire on the Personal Style of the Therapist PST-Q. Clinical Psychology and Psychotherapy, 10, 116-125.

García, F., \& Fernández Álvarez, H. (2007). Investigación empírica sobre el estilo personal del terapeuta: una actualización. Revista Argentina de Clínica Psicológica, 16(2), 121-128.

Henry, W., Schacht, T. \& Strupp, H. (1990). Patient and therapist introject, interpersonal process and differential psychothterapy outcomes. Journal of consulting and clinical psychology, 58, 768-774.

Hernández Sampieri, R., Fernández Collado, C. \& Baptista Lucio, P. (1991). Metodología de la Investigación. México: McGraw.

Huppert, J. D., Bufka, L. F., Barlow, D. H., Gorman, J. M.,Shear, M. K. \& Woods, S. W. (2001). Therapists, therapistsvariables and cognitive behavioral therapy outcome in a multicenter for panic disorder. Journal of Consultingand Clinical Psychology, 69, 747-755.

International Test Commission. (2014). ITC. Retrieved from ITC: http://www. intestcom.org/guidelines/guidelines/users/quality.html

John, O. P., Donahue, E. M., \& Kentle, R. L. (1991). The Big Five InventoryVersions $4 \mathrm{a}$ and 54. Berkeley, CA: University of California, Berkeley, Institute of Personality and Social Research. 
Luborsky, L., McLellan, A. T., Woody, G. E., O’Brien, C. P., \& Auerbach, A. (1985). Therapist success and its determinants. Archives of General Psychiatry, 42, 602-611.

Noftle, E. \& Shaver, P. (2006). Attachment dimensions and the big five personality traits: Associations comparative ability to predict relationship quality. Journal of research in personality, 40, 179-208.

Rial, V., García, F., Castañeiras, C., Gómez, B. \& Fernández Álvarez, H. (2006). Estilo personal del terapeuta que trabajan con pacientes severamente perturbados: un estudio cuanti y cualitativo. Revista de la asociación española de neuropsiquiatría, 2(98), 191-208.

Rubino, G., Barker, C., Roth, T. \& Fearon, P. (2000). Therapist empathy and depth of interaction in response to potencial alliance rupture. Psychotherapy research, 10, 408-420.

Shaver, P. R. \& Brennan, K. A. (1992) Attachment style and the big five personality traits: their connection wirh romantic relationship outcomes. Personality and Social Psychology bulletin, 18, 532-545.

Skourteli, M. \& Lennie, C. (2011). The therapeutic relationship from an attachment theory perspective. Counselling psychology review, 26(1), 20-33. 
